\title{
On Fuzzy Partitions for Visual Texture Modelling
}

\author{
J. Chamorro-Martínez and P. Martínez-Jiménez \\ Department of Computer Science and Artificial Intelligence, \\ University of Granada, Spain \\ email:\{jesus,pedromartinez\}@ decsai.ugr.es
}

\author{
J.M. Soto-Hidalgo \\ Dep. of Comp. Architecture, Electronics and Elect. Tech., \\ University of Córdoba, Spain \\ email: jmsoto@uco.es
}

\begin{abstract}
Texture is one of the most used low-level feature for image analysis and, in addition, one of the most difficult to characterize due to its imprecision. It is usual for humans to describe visual textures according to some perceptual properties like coarseness-fineness, orientation or regularity. In this paper, we propose to model the fineness property, that is the most popular one, by means of a fuzzy partition on the domain of representative fineness measures. In our study, a wide variety of measures is studied, and the partitions are obtained by relating each measure (our reference set) with the human perception of fineness. Assessments about the perception of this property are collected from pools. This information is used to analyze the capability of each measure to discriminate different fineness categories, which imposes the number of fuzzy sets of the partition. Moreover, it is used to calculate the parameters of the membership function associated to each fuzzy set.
\end{abstract}

Index Terms-Coarseness, fineness, fuzzy partition, fuzzy texture, image features, texture features.

\section{INTRODUCTION}

Texture is, together with color and shape, one of the most important features for image analysis. However, it is also one of the most difficult to characterize due to its imprecission [1], [2]. In fact, there is not an accurate definition for the concept of texture but some intuitive ideas, as local changes in the intensity patterns or as a set of basic items arranged in a certain way [3]. Moreover, it is usual for humans to describe visual textures according to some vague textural properties like coarseness/fineness, directionality or regularity [4], [5]. Thus, coarseness is related to the spatial size of texture primitives, directionality reflects if they have a dominant orientation, and regularity refers to the variation of their placement. In this framework, visual textures are described by humans using linguistic terms as fine (e.g. the image in figure $1(\mathrm{~J})$ ), very coarse (figure 1(I)), irregular (figure 1(B)) or very directional (figure 1(I)).

There are many measures in the literature that, given an image, capture the presence of one of these perceptual properties in the sense that the greater the value given by the measure, the greater the perception of the property [3], [4]. However, there is no perceptual relationship between the value given by these measures and the degree in which the humans perceive the texture. Thus, given a certain value calculated by applying a measure to an image, we can not give a textural interpretation (e.g. for coarseness measures, there is not an immediate way to decide whether there is a fine texture, a coarse texture or something intermediate).
To face this problem, fuzzy logic has been recently employed for representing the imprecision related to texture. In many of these approaches, fuzzy logic is usually applied just during the process, being the output a crisp result [6], [7]. Other approaches try to model the texture and its semantic by means of fuzzy sets defined on the domain of a given texture measure. In this last framework, some proposals model the texture property by means of an unique fuzzy set [8], and other approaches define fuzzy partitions providing a set of linguistic terms [9], [10].

Focusing our study in the last type of approaches, two questions need to be faced for defining properly a fuzzy partition: (i) the number of linguistic labels to be used, and (ii) the parameters of the membership functions associated to each fuzzy set (and, consequently, the kernel localization). However, these question are not treat properly in the literature. Firstly, the number of fuzzy sets are often chosen arbitrarily, without take into account the capability of each measure to discriminate between different categories. Secondly, in many of the approaches, just an uniform distribution of the fuzzy sets is performed on the domain of the measures, although is wellknown that measure values corresponding to representative labels are not distributed uniformly. In addition, from our knowledge, none of the fuzzy approaches in the literature considers the relationship between the computational feature and the human perception of texture, so the labels and the membership degrees do not necessarily will match with the human assessments.

Among all the perceptual texture properties, the coarsenessfineness is the most popular one, being considered as the most fundamental feature in texture analysis by some authors [10]. In fact, the presence of fineness is usually associated to the presence of texture (from this point of view, texture is defined as local variations against the idea of homogeneity). In this sense, a fine texture contains small texture primitives with big gray tone differences between neighbor pixels (e.g. the image in Figure 1(A)), whereas a coarse texture corresponds to bigger primitives formed by several pixels (e.g. the image in Figure 1(I)). By considering the importance of the coarseness-fineness concept and in order to explain the methodology proposed in this paper, we will focus our study on this textural property (although other properties can be easily applied in a similar way).

In this paper, we propose to model the fineness property by means of a fuzzy partition on the domain of representative 

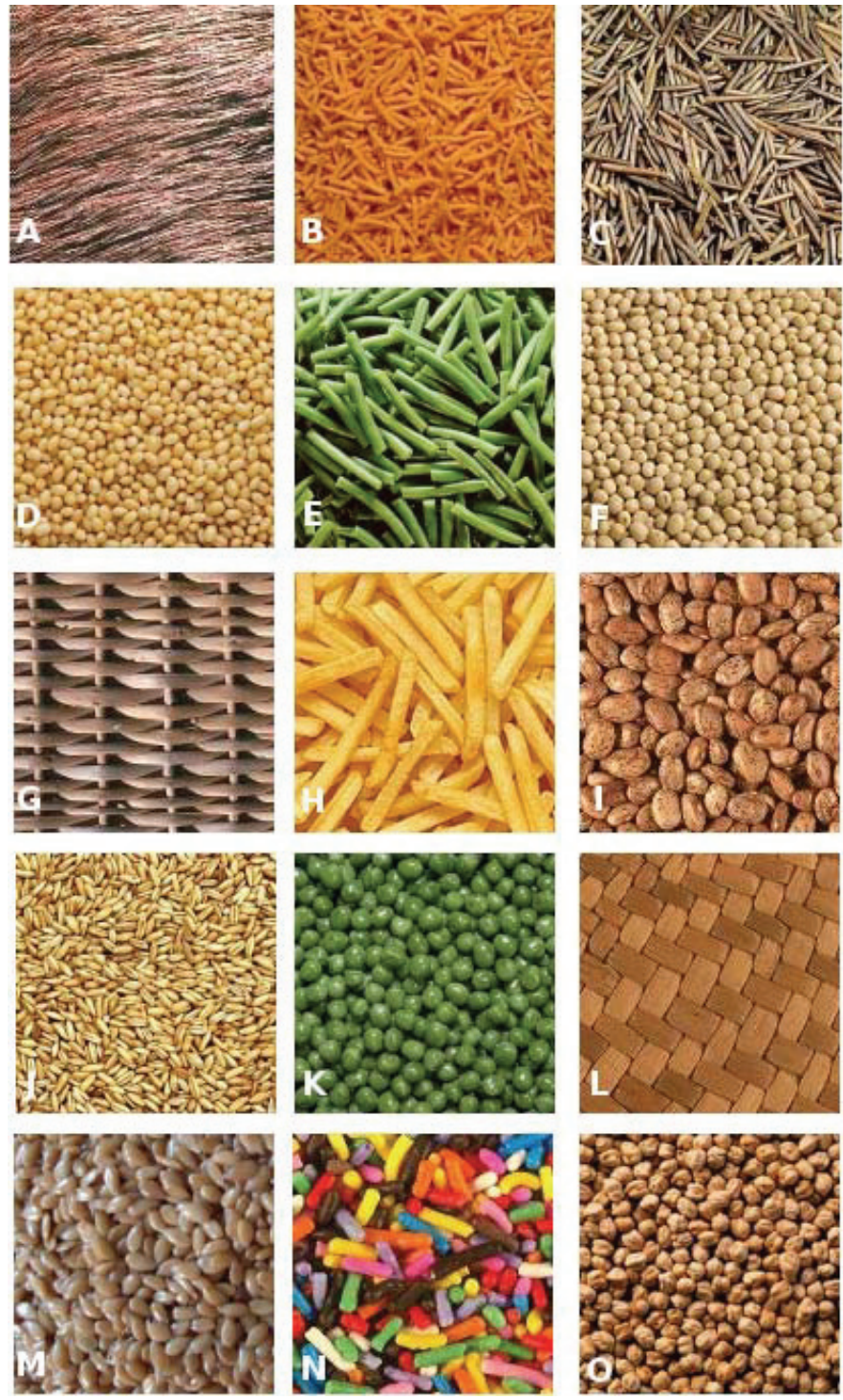

Figure 1. Some examples of images with different degrees of fineness

fineness measures. Firstly, in order to select the number of linguistic labels, we analyze the ability of each measure to discriminate different categories of the perceptual property. For this purpose, data about the human perception of fineness is collected by means of pools. This information is also used to localize the position and size of the kernel of each fuzzy set, obtaining a fuzzy partition adapted to the human perception of fineness.

The rest of the paper is organized as follows. In section II we present our methodology to obtain the fuzzy partition. Results are shown in section III, and the main conclusions and future work are sumarized in section IV.

\section{Defining Fuzzy Partitions for Texture}

Given a texture property (coarseness, directionality, regularity, etc), let $\mathcal{M}$ be a measure of that property and let $\mathcal{D}_{\mathcal{M}}$ be the measure domain. The notions of fuzzy texture and fuzzy texture space are introduced as follows:

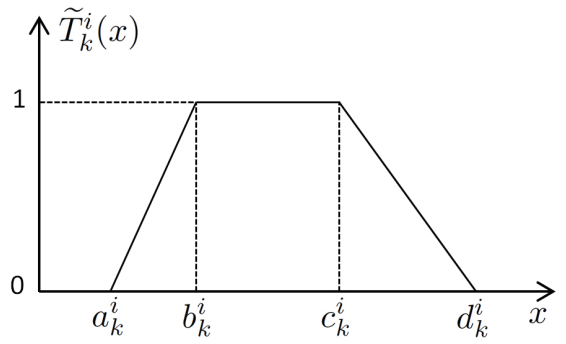

Figure 2. Trapezoidal function used for each fuzzy set $\widetilde{T}_{k}^{i}$

Definition 2.1: A fuzzy texture $\widetilde{T}$ is a linguistic label whose semantics is represented by a normalized fuzzy subset of $\mathcal{D}_{\mathcal{M}}$.

Definition 2.2: A fuzzy texture space $\Pi$ is a set of fuzzy textures that defines a partition of $\mathcal{D}_{\mathcal{M}}$.

In this paper, we will focus our study on the coarsenessfineness property. Thus, we need to define the coarsenessfineness measures $\mathcal{M}$ that are used as the reference set (section II-A).

As it was pointed, there is not a clear perceptual interpretation of the value given by a coarseness-fineness measure. To face this problem, fuzzy partitions on the domain of the measures shown in section II-A are proposed. From now on, we will note $\Pi_{k}$ the partition defined on the domain of a given fineness measure $\mathcal{M}_{k}, N_{k}$ the number of fuzzy sets which compounds the partition $\Pi_{k}$, and $\widetilde{T}_{k}^{i}$ the i-th fuzzy set in $\Pi_{k}$.

In our approach, we propose to define the membership function $\widetilde{T}_{k}^{i}(x)$ for each fuzzy set $\widetilde{T}_{k}^{i}$ by using the trapezoidal function shown in Figure 2, that has the form

$\widetilde{T}_{k}^{i}\left(x ; a_{k}^{i}, b_{k}^{i}, c_{k}^{i}, d_{k}^{i}\right)= \begin{cases}0 & x<a_{k}^{i} \quad \text { or } \quad x>d_{k}^{i} \\ \frac{x-a_{k}^{i}}{b_{k}^{i}-a_{k}^{i}} & a_{k}^{i} \leq x \leq b_{k}^{i} \\ 1 & b_{k}^{i} \leq x \leq c_{k}^{i} \\ \frac{d_{k}^{i}-x}{d_{k}^{k}-c_{k}^{i}} & c_{k}^{i} \leq x \leq d_{k}^{i}\end{cases}$

This way, two questions need to be faced in order to define the fuzzy partition $\Pi_{k}$ : (i) how many fuzzy sets will compound the partition, and (ii) how to obtain the parameter values of the membership function for each fuzzy set.

In order to answer both questions, we will analyze the ability of each measure to distinguish between different degrees of fineness. This analysis will be based on how the human perceives the fineness. To get information about human perception of fineness, a set of images covering different degrees of this property will be gathered. These images will be used to collect, by means of a pool, human assessments about the perceived fineness (section II-B)

Using the data about human perception, and the measure values obtained for each image, we will apply an iterative algorithm based on a set of multiple comparison tests, in order to obtain the number of classes (fineness degrees) that each measure can discriminate. Thus, we propose to set the number 


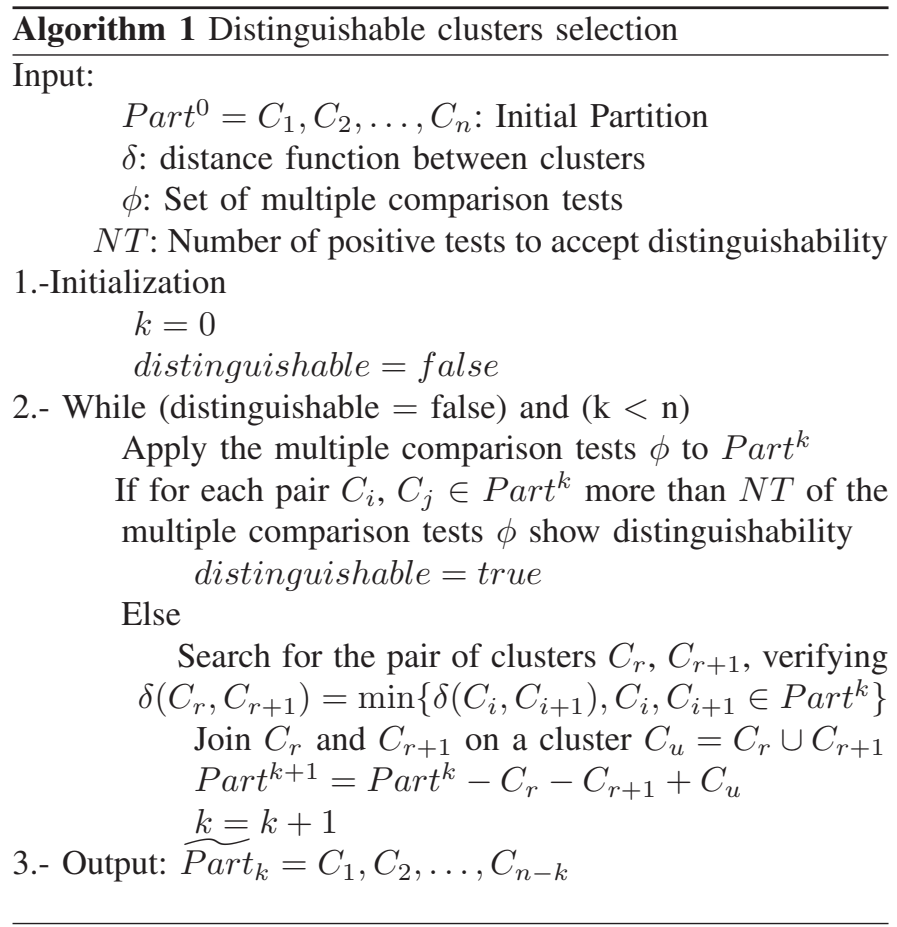

of fuzzy sets $N_{k}$ in the partition $\Pi_{k}$ as the number of classes that can be discriminated by the measure $\mathcal{M}_{k}$ (section II-C). In addition, the information given by the tests, will be used to define the parameter values of the membership function $\widetilde{T}_{k}^{i}(x)$ for each fuzzy set $\widetilde{T}_{k}^{i}$ (section II-D).

\section{A. Texture measures}

As mentioned in the introduction, there are many measures in the literature that, given an image, capture the fineness (or coarseness) presence in the sense that the greater the value given by the measure, the greater the perception of texture [4], [3]. In this paper, some of the most representative of these measures, that are listed in the first column of Table I, have been analyzed. It should be noticed that most of these measures were developed in the early nineties. However, they are still considered the state of the art in the characterization of the coarseness property of textures. In fact, they are achieving a growing interest due to applications in fields like image retrieval, where low level features like texture are the basis for the description of visual content (e.g. MPEG-7 descriptors) [11], [12], or for learning new concepts [13], [14]. All the coarseness-fineness measures analyzed in this paper are automatically computed from the texture image, obtaining a real value. Thus, the domain for all of them is $\mathcal{D}_{\mathcal{M}}=\mathbb{R}$.

\section{B. Assessment collection}

From now on, let $\mathcal{I}=\left\{I_{1}, \ldots, I_{N}\right\}$ be the set of $N$ images representing fineness examples, and let $\Gamma=\left\{v^{1}, \ldots, v^{N}\right\}$ be the set of perceived fineness values associated to $\mathcal{I}$, with $v^{i}$ being the value representing the degree of fineness perceived by humans in the image $I_{i} \in \mathcal{I}$. In this section, the way to obtain the set $\Gamma$ will be described. For this purpose, firstly the image set $\mathcal{I}$ will be selected (section II-B1). After that, a poll for getting assessments about the perception of fineness will be designed (section II-B2). Finally, for a given image, the assessments of the different subjects will be aggregated (section II-B3).

1) The texture image set: A set $\mathcal{I}=\left\{I_{1}, \ldots, I_{N}\right\}$ of $N=$ 80 images representative of the concept of fineness has been selected. Figure 1 shows some images extracted from the set $\mathcal{I}$. Such set has been selected satisfying the following properties:

1) It covers the different presence degrees of fineness.

2) The number of images for each presence degree is representative enough.

3) Each image shows, as far as possible, just one presence degree of fineness.

Due to the third property, each image can viewed as "homogeneous" respect to the fineness degree represented, i.e., if we select two random windows (with a dimension which does not "break" the original texture primitives and structure), the perceived fineness will be the same for each window (and also respect to the original image). In other words, we can see each image $I_{i} \in \mathcal{I}$ as a set of lower dimension images (windows) with the same fineness degree of the original one.

As we explained, given an image $I_{i} \in \mathcal{I}$, a fineness measure $\mathcal{M}_{k}$ will be applied on it. In fact, and thanks to the third property, we really can apply this measure for each subimage, assuming that the human assessment associated to that subimage will be the human assessment associated to the whole image. From now on, we will note as $m_{k}^{i, w}$ the result of applying the measure $\mathcal{M}_{k}$ to the $w$-th window of the image $I_{i}$.

2) The poll: Given the image set $\mathcal{I}$, the next step is to obtain assessments about the perception of fineness from a set of subjects. From now on we shall note as $\Theta^{i}=\left[o_{1}^{i}, \ldots, o_{L}^{i}\right]$ the vector of assessments obtained from $L$ subjects for the image $I_{i}$. To get $\Theta^{i}$, subjects will be asked to assign images to classes, so that each class has associated a perception degree of fineness. In particular, 20 subjects have participated in the poll and 9 classes have been considered. The first nine images in figure 1 show the nine representative images for each class used in this poll. It should be noticed that the images are decreasingly ordered according to the degree of fineness.

As result, a vector of 20 assessments $\Theta^{i}=\left[o_{1}^{i}, \ldots, o_{20}^{i}\right]$ is obtained for each image $I_{i} \in \mathcal{I}$. The degree $o_{j}^{i}$ associated to the assessment given by the subject $S_{j}$ to the image $I_{i}$ is computed as $o_{j}^{i}=(9-k) * 0.125$, where $k \in\{1, \ldots, 9\}$ is the index of the class $C_{k}$ to which the image is assigned by the subject.

3) Assessment aggregation: Our aim at this point is to obtain, for each image in the set $\mathcal{I}$, one assessment $v^{i}$ that summarizes the assessments $\Theta^{i}$ given by the different subjects about the presence degree of fineness.

To aggregate opinions we have used an OWA operator guided by a quantifier [24]. Concretely, the quantifier "the most" has been employed, which allows to represent the opinion of the majority of the polled subjects. This quantifier is defined as 
Table I

RESULT OBTAINED BY APPLYING THE ALGORITHM 1

\begin{tabular}{|l|c|c|c|c|c|c|c|}
\hline Measure & $N_{k}$ & Classes & $\bar{c}_{5} \pm \Psi_{5}$ & $\bar{c}_{4} \pm \Psi_{4}$ & $\bar{c}_{3} \pm \Psi_{3}$ & $\bar{c}_{2} \pm \Psi_{2}$ & $\bar{c}_{1} \pm \Psi_{1}$ \\
\hline Correlation [3] & 5 & $\{1,2-4,5-6,7-8,9\}$ & $0.122 \pm 0.038$ & $0.403 \pm 0.0272$ & $0.495 \pm 0.0225$ & $0.607 \pm 0.0133$ & $0.769 \pm 0.0210$ \\
ED [15] & 5 & $\{1,2,3-5,6-8,9\}$ & $0.348 \pm 0.0086$ & $0.282 \pm 0.0064$ & $0.261 \pm 0.0063$ & $0.238 \pm 0.0066$ & $0.165 \pm 0.0061$ \\
Abbadeni [16] & 4 & $\{1,2-6,7-8,9\}$ & - & $5.672 \pm 0.2738$ & $9.208 \pm 0.4247$ & $11.12 \pm 0.2916$ & $25.23 \pm 1.961$ \\
Amadasun [4] & 4 & $\{1,2-6,7-8,9\}$ & - & $4.864 \pm 0.271$ & $7.645 \pm 0.413$ & $9.815 \pm 0.230$ & $19.62 \pm 1.446$ \\
Contrast [3] & 4 & $\{1,2-5,6-8,9\}$ & - & $3312 \pm 265.5$ & $2529 \pm 295.5$ & $1863 \pm 94.84$ & $790.8 \pm 129.4$ \\
FD [17] & 4 & $\{1,2,3-8,9\}$ & - & $3.383 \pm 0.0355$ & $3.174 \pm 0.0282$ & $2.991 \pm 0.0529$ & $2.559 \pm 0.0408$ \\
Tamura [5] & 4 & $\{1,2-6,7-8,9\}$ & - & $1.540 \pm 0.0634$ & $1.864 \pm 0.0722$ & $2.125 \pm 0.0420$ & $3.045 \pm 0766$ \\
Weszka [18] & 4 & $\{1,2-6,7-8,9\}$ & - & $0.153 \pm 0.0064$ & $0.113 \pm 0.0093$ & $0.099 \pm 0.0036$ & $0.051 \pm 0.0041$ \\
DGD [19] & 3 & $\{1,2-8,9\}$ & - & - & $0.020 \pm 0.0010$ & $0.038 \pm 0.0017$ & $0.091 \pm 0.0070$ \\
FMPS [20] & 3 & $\{1,2-8,9\}$ & - & - & $0.256 \pm 0.0477$ & $0.138 \pm 0.0122$ & $0.0734 \pm 0.0217$ \\
LH [3] & 3 & $\{1,2-8,9\}$ & - & - & $0.023 \pm 0.0010$ & $0.052 \pm 0.0025$ & $0.127 \pm 0.0096$ \\
Newsam [21] & 3 & $\{1,2-6,7-9\}$ & - & - & $0.1517 \pm 0.0425$ & $0.2654 \pm 0.0466$ & $0.4173 \pm 0.0497$ \\
SNE [22] & 3 & $\{1,2-8,9\}$ & - & - & $0.879 \pm 0.0182$ & $0.775 \pm 0.0087$ & $0.570 \pm 0.0232$ \\
SRE [23] & 3 & $\{1,2-8,9\}$ & - & - & $0.995 \pm 0.00026$ & $0.987 \pm 0.00066$ & $0.966 \pm 0.0030$ \\
Entropy [3] & 2 & $\{1,2-9\}$ & - & - & - & $9.360 \pm 0.124$ & $8.656 \pm 0.301$ \\
Uniformity[3] & 2 & $\{1,2-9\}$ & - & - & - & $1.3 E^{-4} \pm 2.6 E^{-5}$ & $3.9 E^{-4} \pm 1.9 E^{-4}$ \\
Variance[3] & 1 & - & - & - & - & - \\
\hline
\end{tabular}
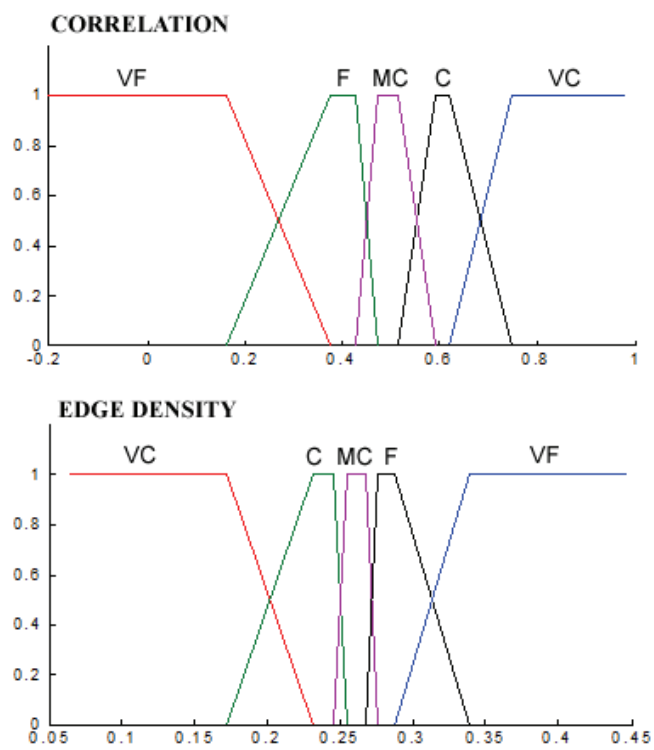

Figure 3. Fuzzy partitions for the measures Correlation and Edge Density. The linguistic labels are $\mathrm{VC}=$ very coarse, $\mathrm{C}=$ coarse, $\mathrm{MC}=$ medium coarse, $\mathrm{F}=$ fine, $\mathrm{VF}=$ very fine

$$
Q(r)=\left\{\begin{array}{lr}
0 & \text { if } r<a \\
\frac{r-a}{b-a} & \text { if } a \leq r \leq b, \\
1 & \text { if } r>b
\end{array}\right.
$$

with $r \in[0,1], a=0.3$ and $b=0.8$. Once the quantifier $Q$ has been chosen, the weighting vector of the OWA operator can be obtained following Yager [24] as $w_{j}=Q(j / L)-Q((j-$ $1) / L), j=1,2, \ldots, L$. According to this, for each image $I_{i} \in$ $\mathcal{I}$, the vector $\Theta^{i}$ obtained from $L$ subjects will be aggregated into one assessment $v^{i}$ as follows:

$$
v^{i}=w_{1} \hat{o}_{1}^{i}+w_{2} \hat{o}_{2}^{i}+\ldots+w_{L} \hat{o}_{L}^{i}
$$

where $\left[\hat{o}_{1}^{i}, \ldots, \hat{o}_{L}^{i}\right]$ is a vector obtained by ranking in nonincreasing order the values of the vector $\Theta^{i}$.

\section{Distinguishability Analysis of the Fineness Measures}

As it was expected, some measures have better ability to represent fineness than the others. To study the ability of each measure to discriminate different degrees of fineness (i.e. how many classes can $\mathcal{M}_{k}$ actually discriminate), we propose to analyze each measure by applying a set of multiple comparison tests following the algorithm 1. This algorithm starts with an initial partition ${ }^{1}$ and iteratively joins clusters until a partition in which all classes are distinguishable is achieved. In our proposal, the initial partition will be formed by the 9 classes used in our poll (where each class will contain the images assigned to it by the majority of the subjects), as $\delta$ the Euclidean distance between the centroids of the involved classes will be used, as $\phi$ a set of 5 multiple comparison tests will be considered (concretely, the tests of Scheffé, Bonferroni, Duncan, Tukey's least significant difference, and Tukey's honestly significant difference [25]), and finally the number of positive tests to accept distinguishability will be fixed to $N T=3$.

From now on, we shall note as $\Upsilon_{k}=C_{1}^{k}, C_{2}^{k}, \ldots, C_{N_{k}}^{k}$ the $N_{k}$ classes that can be discriminated by $\mathcal{M}_{k}$. For each $C_{i}^{k}$, we will note as $\bar{c}_{i}^{k}$ the class representative value. In this paper, we propose to compute $\bar{c}_{i}^{k}$ as the mean of the measure values in the class $C_{i}^{k}$.

Table I shows the parameters obtained by applying the proposed algorithm with the different measures considered in this paper. The second column of this table shows the $N_{k}$ classes that can discriminate each measure and the third column shows how the initial classes have been grouped. The columns from fourth to eighth show the representative values $\bar{c}_{r}^{k}$ associated to each cluster.

\section{The Fuzzy Partitions}

In this section we will deal with the problem of defining the membership function $\mathcal{T}_{k}^{i}(x)$ for each fuzzy set $\mathcal{T}_{k}^{i}$ compounding the partition $\Pi_{k}$. As it was explained, the number of

\footnotetext{
${ }^{1}$ Let us remark that this partition is not the "fuzzy partition". In this case, the elements are measure values and the initial clusters the ones given by the pool.
} 
Table II

PARAMETER VALUES THAT DEFINE THE MEMBERSHIP FUNCTIONS $\mathcal{T}_{k}^{i}(x)$ COMPOUNDING THE PARTITION FOR THE MEASURES OF CORRELATION AND EDGE DENSITY (a)

\begin{tabular}{ccccc}
\multicolumn{5}{c}{ CORRELATION } \\
\hline $\mathrm{i}$ & $a_{k}^{i}$ & $b_{k}^{i}$ & $c_{k}^{i}$ & $d_{k}^{i}$ \\
\hline 1 & $-\infty$ & $-\infty$ & 0.1600 & 0.3758 \\
2 & 0.1600 & 0.3758 & 0.4302 & 0.4725 \\
3 & 0.4302 & 0.4725 & 0.5175 & 0.5937 \\
4 & 0.5175 & 0.5937 & 0.6203 & 0.7480 \\
5 & 0.6203 & 0.7480 & $\infty$ & $\infty$ \\
\hline
\end{tabular}

(b)

\begin{tabular}{ccccc}
\multicolumn{5}{c}{ EDGE DENSITY } \\
\hline $\mathrm{i}$ & $a_{k}^{i}$ & $b_{k}^{2}$ & $c_{k}^{i}$ & $d_{k}^{i}$ \\
\hline 1 & $-\infty$ & $-\infty$ & 0.1711 & 0.2314 \\
2 & 0.1711 & 0.2314 & 0.2446 & 0.2547 \\
3 & 0.2446 & 0.2547 & 0.2673 & 0.2756 \\
4 & 0.2673 & 0.2756 & 0.2884 & 0.3394 \\
5 & 0.2884 & 0.3394 & $\infty$ & $\infty$ \\
\hline
\end{tabular}

fuzzy sets will be given by the number of categories that each measure can discriminate (shown in Table I).

In this paper, the trapezoidal function given by equation (1) is used for defining each membership function $\widetilde{T}_{k}^{i}(x)$. In addition, a fuzzy partition in the sense of Ruspini is proposed. Figure 3 shows some examples of the type of fuzzy partition used.

To establish the value of the parameters $b_{k}^{i}$ and $c_{k}^{i}$, that define the kernel of the trapezoidal membership function associated to the fuzzy set $\widetilde{T}_{k}^{i}$, we propose a solution based on the multiple comparison tests used in section II-C. As it is known, in these tests confidence intervals around the representative value of each class are calculated (being accomplished that these intervals do not overlap for distinguishable classes). All values in the interval are considered plausible values for the estimated mean. These confidence intervals are defined by the parameter $\Psi_{i}^{k}=1.96 \bar{\sigma}_{i}^{k} / \sqrt{\left\|C_{i}^{k}\right\|}$, with $\bar{c}_{i}^{k}$ and $\bar{\sigma}_{i}^{k}$ being the representative value and the estimated standard deviation for the class $C_{i}^{k}$, respectively. Table I shows the values $\Psi_{i}^{k}$ for each measure and each class.

Based on this idea, we propose to set the center of the kernel of the membership function $\widetilde{T}_{k}^{i}(x)$ as the representative value $\bar{c}_{i}^{k}$ of the corresponding i-th class, using the information of the confidence interval to obtain the value of the parameters $b_{k}^{i}$ and $c_{k}^{i}$ :

$$
b_{k}^{i}=\bar{c}_{i}^{k}-\Psi_{i}^{k} \quad c_{k}^{i}=\bar{c}_{i}^{k}+\Psi_{i}^{k}
$$

Since a fuzzy partition in the sense of Ruspini is proposed, the value of the parameters $a_{k}^{i}$ and $d_{k}^{i}$ is obtained as

$$
a_{k}^{i}=c_{k}^{i-1} \quad d_{k}^{i}=b_{k}^{i+1}
$$

It should be noticed that $a_{k}^{1}=b_{k}^{1}=-\infty$ and $c_{k}^{N_{k}}=d_{k}^{N_{k}}=$ $\infty$.

Figure 3 shows the graphical representation of the fuzzy partitions for the measures of correlation and ED (the ones with higher capacity to discriminate fineness classes), and Table II shows their corresponding parameters.

\section{RESULTS}

In this section, the fuzzy partition defined for the measure "Correlation" (showed in Figure 3) will be applied in order to analyze the performance of the proposed model.

Let's consider Figure 4(a) corresponding to a mosaic made by several images, each one with a different increasing degree (a)

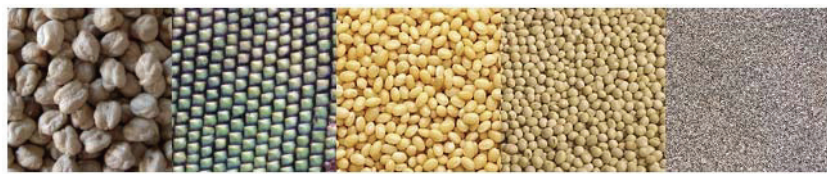

(b)

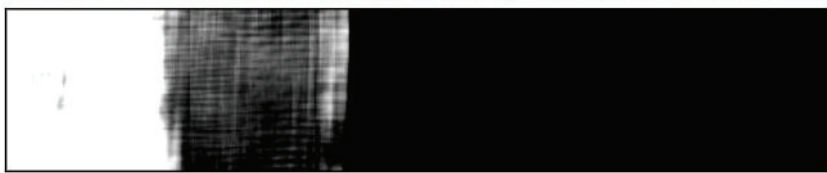

(c)

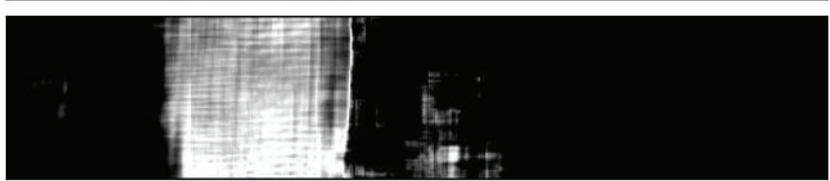

(d)

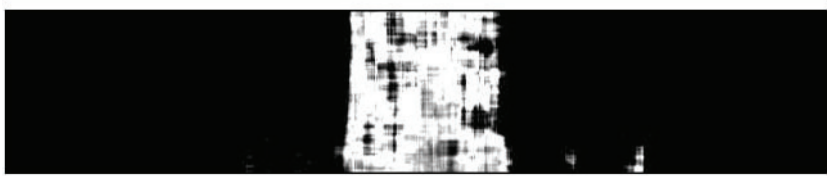

(e)

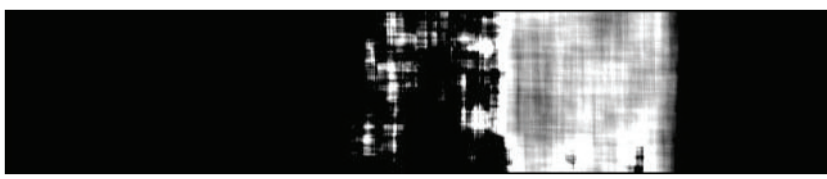

(f)

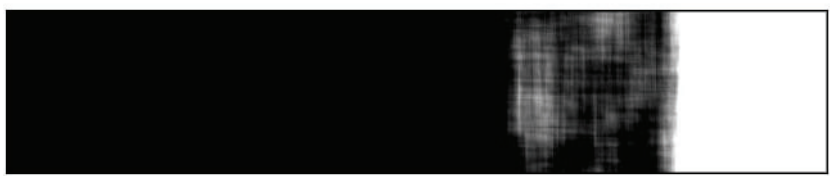

Figure 4. Results for a mosaic image. (a) Original image (b)(c)(d)(e)(f) Membership degree of each pixel to the sets "very coarse", "coarse", "medium coarse", "fine" and "very fine", respectively (the darker the pixel, the lower the membership degree).

of fineness. Figure 4(b-f) shows the membership degree to the fuzzy sets "very coarse", "coarse", "medium coarse", "fine" and "very fine", respectively, using the proposed model. For each pixel in the original image, a centered window of size $32 \times 32$ has been analyzed and its membership degree to each fuzzy set has been calculated. Thus, Figure 4(b) represents the degree in which the texture is perceived as "very coarse", with a white level meaning maximum degree, and a dark one meaning zero degree. It can be noticed that our model captures the evolution of the perception degrees of fineness.

Figure 5 presents an example where the proposed fuzzy partition has been employed for pattern recognition. In this case, Figure 5(a) shows a microscopy image corresponding to the microstructure of a metal sample. The lamellae indicates islands of eutectic, which are to be separated from the uniform light regions. The brightness values in regions of the original image are not distinct, so texture information is needed for extracting the uniform areas. This fact is showed in Figures 5(b) and 5(c), where a thersholding on the original image is displayed (homogeneous regions cannot be separated from the textured ones as they "share" brightness values). Figure 5(d) shows a mapping from the original image to its membership degree to the fuzzy set associated "very coarse". Thus, Figure 5(d) represents the degree in which the texture is perceived as "very coarse" and it can be noticed that uniform regions 


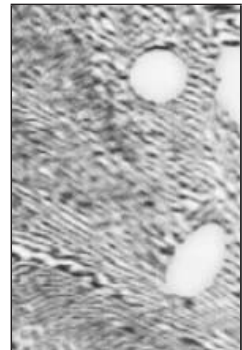

(a)

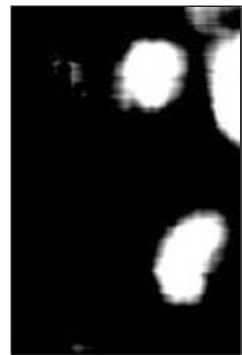

(d)

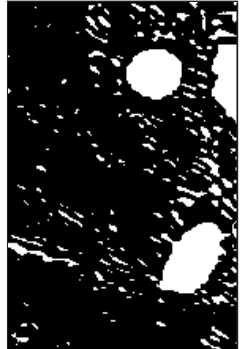

(b)

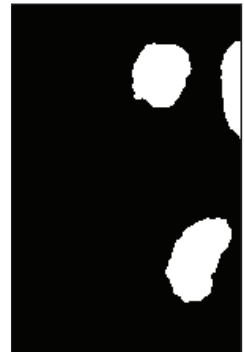

(e)

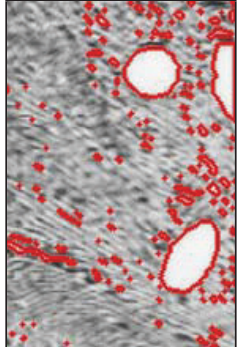

(c)

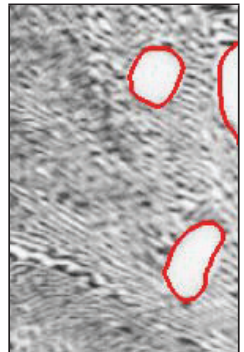

(f)
Figure 5. Example of pattern recognition (a) Original image (b) Binary image obtained by thresholding the original one (c) Region outlines of $b$ superimposed on original image (d) Membership degrees to the set "very coarse" obtained with our model from the original image (e) Binary image obtained by thresholding $d$ (f) Region outlines of $e$ superimposed on original image.

correspond to areas with the maximum degree (bright grey levels), so if only the pixels with degree upper than 0.9 are selected (Figure 5(e)), the uniform light regions emerge with ease (Figure 5(f)).

Figure 6 presents another example of the application of the proposed fuzzy partition for pattern recognition. Figure 6(a) shows the natural image used in this example, that corresponds to a microscopic image of a Volvox (a colony of greencells). In this case, we want to separate the main colony from the background and the daughter colonies from the mother colony. As in the previous example, this can not be done by thresholding on the original image, as it is shown in Figure 6(b), so texture information is needed. Figures 6(c), 6(d) and 6(e) shows the mapping from the original image to its membership degree to the fuzzy sets "very fine", "medium coarse" and "very coarse", respectively. It can be noticed that daughter colonies in Figure 6(a) correspond to areas with high degrees of fineness, so they can be obtained by selecting the pixels with membership degree higher than 0.9 to the fuzzy set "very fine". On the other hand, the main colony can be separated from the uniform background as in the previous example (with a threshold of 0.9 in the membership degree to the fuzzy set "very coarse"), obtaining the region outlines shown in Figure 6(f)

\section{CONCLUSIONS AND FUTURE WORKS}

In this paper, a fuzzy partition for representing the fineness concept have been proposed. The number of fuzzy sets and

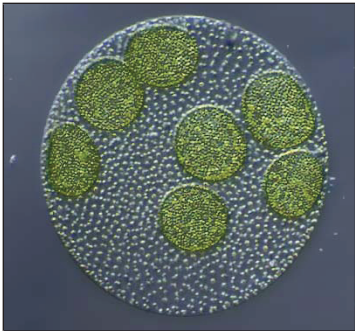

(a)

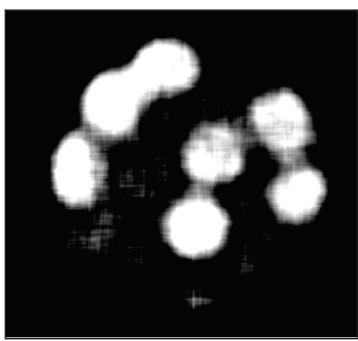

(c)

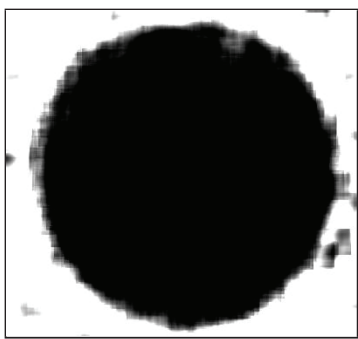

(e)

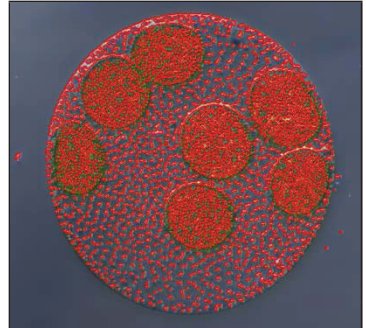

(b)

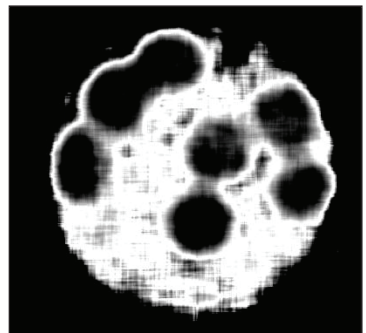

(d)

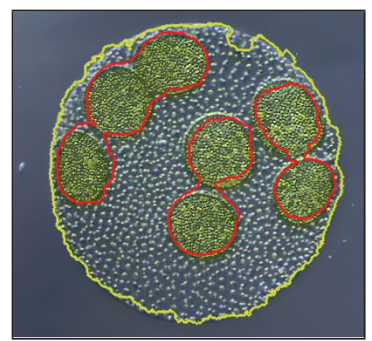

(f)
Figure 6. Results for a microscopy image. (a) Original image (b) Thresholding performed on $a$ superimposed on original image (c)(d)(e) Membership degrees to the sets "very fine", "medium coarse" and "very coarse", respectively (f) Thresholding performed on $c$ and $e$ superimposed on original image.

the parameters of the membership functions have been defined relating fineness measures with the human perception of this texture property. Pools have been used for collecting data about the human perception of fineness, and the capability of each measure to discriminate different fineness degrees has been analyzed. The results given by our approach show a high level of connection with the human perception of fineness. As future work, the performance of the fuzzy partition will be analyzed in applications like textural classification or segmentation.

\section{ACKNOWLEDGMENT}

This work has been partially supported by the Spanish Government under the TIN2009-08296 and Consolider Ingenio 2010 MIPRCV (CSD2007-00018) projects.

\section{REFERENCES}

[1] C. Jacobs, A. Finkelstein, and D. Salesin, "Fast multiresolution image querying," in Proc. Computer Graphics, Annual Conference Series, 1995, pp. 277-286.

[2] S. Belongie, C. Carson, H. Greenspan, and J. Malik, "Color- and texturebased image segmentation using expectation-maximization and its application to content-based image retrieval," in Proc. 6th International Conference on Computer Vision, 1998, pp. 675-682. 
[3] R. Haralick, "Statistical and structural approaches to texture," Proceedings IEEE, vol. 67, no. 5, pp. 786-804, 1979.

[4] M. Amadasun and R. King, "Textural features corresponding to textural properties," IEEE Transactions on Systems, Man and Cybernetics, vol. 19, no. 5, pp. 1264-1274, 1989.

[5] H. Tamura, S. Mori, and T. Yamawaki, "Textural features corresponding to visual perception," IEEE Trans. on Systems, Man and Cybernetics, vol. 8, pp. 460-473, 1978.

[6] M. Hanmandlu, V. K. Madasu, and S. Vasikarla, "A fuzzy approach to texture segmentation," in Proc. International Conference on Information Technology: Coding and Computing, vol. 1, 2004, pp. 636-642.

[7] A. Barcelo, E. Montseny, and P. Sobrevilla, "Fuzzy texture unit and fuzzy texture spectrum for texture characterization," Fuzzy Sets and Systems, vol. 158, pp. 239-252, 2007.

[8] J. Chamorro-Martinez, E. Galan-Perales, J. Soto-Hidalgo, and B. PradosSuarez, "Using fuzzy sets for coarseness representation in texture images," in Proceedings IFSA'07, 2007, pp. 783-792.

[9] S. Kulkarni and B. Verma, "Fuzzy logic based texture queries for cbir," in Proc. 5th International Conference on Computational Intelligence and Multimedia Applications, 2003, pp. 223-228.

[10] H. Lin, C. Chiu, and S. Yang, "Finding textures by textual descriptions, visual examples, and relevance feedbacks," Pattern Recognition Letters, vol. 24, no. 14, pp. 2255-2267, 2003.

[11] P. Wu, Y. M. Ro, C. S. Won, and Y. Choi, "Texture descriptors in mpeg7," in Proceedings of the 9th International Conference on Computer Analysis of Images and Patterns, ser. CAIP '01. Springer-Verlag, 2001, pp. 21-28.

[12] B. S. Manjunath, Introduction to MPEG-7, Multimedia Content Description Interface, P. S. B. S. Manjunath and T. Sikora, Eds. John Wiley and Sons, Ltd., Jun 2002.

[13] B. Manjunath, J.-R. Ohm, V. Vasudevan, and A. Yamada, "Color and texture descriptors," Circuits and Systems for Video Technology, IEEE Transactions on, vol. 11, no. 6, pp. 703 -715, June 2001.

[14] H. Lin, C. Chiu, and S. Yang, "Finding textures by textual descriptions, visual examples, and relevance feedbacks," Pattern Recogn. Lett., vol. 24, pp. 2255-2267, October 2003.

[15] J. Canny, "A computational approach to edge detection," IEEE Transactions on Pattern Analysis and Machine Intelligence, vol. 8, no. 6, pp. 679-698, 1986.

[16] N. Abbadeni, N. Ziou, and D. Wang, "Autocovariance-based perceptual textural features corresponding to human visual perception," in Proc. of 15th International Conference on Pattern Recognition, vol. 3, 2000, pp. 901-904.

[17] S. Peleg, J. Naor, R. Hartley, and D. Avnir, "Multiple resolution texture analysis and classification," IEEE Transactions on Pattern Analysis and Machine Intelligence, no. 4, pp. 518-523, 1984.

[18] J. Weszka, C. Dyer, and A. Rosenfeld, "A comparative study of texture measures for terrain classification," IEEE Trans. on SMC, vol. 6, pp. 269-285, 1976.

[19] S. Kim, K. Choi, and D. Lee, "Texture classification using run difference matrix," in Proc. of IEEE 1991 Ultrasonics Symposium, vol. 2, December 1991, pp. 1097-1100.

[20] H. Yoshida, D. Casalino, B. Keserci, A. Coskun, O. Ozturk, and A. Savranlar, "Wavelet-packet-based texture analysis for differentiation between benign and malignant liver tumours in ultrasound images," Physics in Medicine and Biology, vol. 48, pp. 3735-3753, 2003.

[21] S. Newsam and C. Kammath, "Retrieval using texture features in high resolution multi-spectral satellite imagery," in Data Mining and Knowledge Discovery: Theory, Tools, and Technology VI, SPIE Defense and Security, April 2004.

[22] C. Sun and W. Wee, "Neighboring gray level dependence matrix for texture classification," Computer Vision, Graphics and Image Processing, vol. 23, pp. 341-352, 1983.

[23] M. Galloway, "Texture analysis using gray level run lengths," Computer Graphics and Image Processing, vol. 4, pp. 172-179, 1975.

[24] R. Yager, "On ordered weighted averaging aggregation operators in multicriteria decisionmaking," IEEE Trans. on SMC, vol. 18, no. 1, pp. 183-190, 1988.

[25] Y. Hochberg and A. Tamhane, Multiple Comparison Procedures. Wiley, 1987. 